\title{
Traditional chinese medicine diet paratherapy for alleviating toxicity in chemotherapy and radiotherapy in cancer patients: a meta-analysis
}

\author{
Xiaoying ZHOU ${ }^{1}\left(\mathbb{0}\right.$, Guoying WANG ${ }^{1}$, Lei LAI ${ }^{1}$, Li XU ${ }^{1}$, Qiuping SHEN ${ }^{1}$, Yingjie WANG ${ }^{1}$, Mingzhu FAN ${ }^{1}$, Liping SHEN ${ }^{1 *}$
}

\begin{abstract}
The aim of this meta-analysis was to evaluate the efficacy of Traditional Chinese Medicine (TCM) diet therapy in alleviating the toxicity of chemotherapy and radiotherapy in patients with cancer. Searches were conducted in seven databases for relevant studies published from 1, January 2000 to 1 March, 2020. Randomized controlled trials (RCTs) comparing TCM diet with ordinary diet of cancer patients undergoing chemotherapy and(or) radiotherapy were included. The study has been registered on the "PROSPERO" website with registration number of CRD42019131232. A total of sixteen RCTs included 2336 cases were included. TCM diet therapy is superior to ordinary diet in terms of reducing incidence of leukopenia $[\mathrm{RR}=0.57 ; 95 \% \mathrm{CI}(0.47$, $0.69) ; \mathrm{P}<0.0001]$, and nausea and vomiting $[\mathrm{RR}=0.54,95 \% \mathrm{CI}=(0.43,0.70), \mathrm{P}=0.004]$, increasing hemoglobin $[\mathrm{MD}=10.04$; 95\% CI (7.66, 12.41); $\mathrm{P}<0.0001]$, improving Karnofsky Performance Status (KPS) [RR=1.28; 95\% CI $(1.15,1.42) ; \mathrm{P}<0.0001]$, and gaining weight $[\mathrm{RR}=1.53,95 \% \mathrm{CI}=(1.38,1.68), \mathrm{P}<0.0001]$ in patients with cancer undergoing chemotherapy and(or) radiotherapy. This meta-analysis demonstrated that TCM diet may reduce the toxicity of chemotherapy (and/or radiotherapy).
\end{abstract}

Keywords: Traditional Chinese medicine diet; meta-analysis; paratherapy; alleviate toxicity; randomized controlled trials; chemotherapy; radiotherapy.

Practical Application: The efficacy of Traditional Chinese Medicine (TCM) diet therapy in alleviating the toxicity of chemotherapy and radiotherapy in patients with cancer

\section{Introduction}

In 2018, the newly diagnosed cancer cases were 18.1 million, and the deaths from cancer were about 9.6 million (Bray et al., 2018). The standard therapies of cancer, such as chemotherapy, radiotherapy, surgery, immunotherapy, targeted therapy, have different levels of side effects. Therefore, a lot of patients were unable to complete the entire therapy due to serious side effects especially after chemotherapy, or radiotherapy. What's more, it would be a marked impact on quality of life. Therefore, effective solution is needed to alleviate the toxicity. At present, most guidelines recommend symptomatic treatment of the side effects such as vomiting and nausea with 5-hydroxytryptamine (5-HT3, serotonin) receptor antagonists, tachykinin NK1 receptor antagonist, steroids, olanzapine, dopamine receptor antagonists, and benzodiazepines (Jordan et al., 2014). However, complete control of chemotherapy-induced nausea and vomiting (CINV) remains unsolved (Hsieh et al., 2015). The side effects of bone marrow depression were treated with therapy such as colonystimulating factor or interleukin-11 (Crawford et al., 1991; Nemunaitis et al., 1991), but additional interventions were still needed to deal with the adverse effects. It was reported that Traditional Chinese medicine (TCM) could be beneficial in alleviating the side effects (Nie et al., 2016). TCM diet is an important part of TCM treatment, and the relevant diets or combining foods with herbs are purposefully chosen for the

patients by syndrome, in order to balance the yin and yang, and harmonize qi and blood (Wu \& Liang, 2018).

A study has shown that TCM diet may reduce adverse reactions such as nausea and vomiting after chemotherapy, and also could improve the quality of life of the patients (Zhao et al., 2017). The corresponding dietetic and nutrition recommendations are formulated according to the TCM nutritional treatment for colorectal cancer in China and the diagnosis and treatment standard of TCM syndrome differentiation for colorectal cancer in the "Guide to TCM Diagnosis and Treatment of Malignant Tumors" (Li et al., 2017).

A number of clinical studies have been published in China in recent years. Six studies (Chang et al., 2011; Huang et al., 2011; Wang et al., 2011, 2012; Shi et al., 2012; Zhang, 2009) reported that the treatment groups significantly attenuated leukopenia in contrast to the control groups. Five studies (Ao, 2013; Pan \& Chen, 2001; Yue et al., 2007; Zhang et al., 2014; Liu et al., 2015) reported higher hemoglobin in the experimental group than that in the control group. Nine studies (Chang et al., 2011; Wang et al., 2011; Zhang, 2009; Yue et al., 2007; Zhang et al., 2014; Liu et al., 2015; Fang et al., 2018; Qu, 2018; Jiang et al., 2015) reported the incidence of nausea and vomiting, and made comparison between TCM diet and ordinary diet. TCM diet was suggested to be more effective in symptoms relief. However, none of these comprehensively 
reviewed the effects of TCM diet therapy in alleviating toxicity and side effects after radiotherapy and chemotherapy. As far as we know, this meta-analysis was the first to evaluate the effects of TCM diet therapy in alleviating the toxicity in cancer patients undergoing chemotherapy (and/or radiotherapy).

\section{Materials and methods}

\subsection{Standard protocol registrations}

This meta-analysis is registered in PROSPERO, registration ID: CRD 42019131232 and the protocol has been published. The procedure of this study is strictly based on the Preferred Reporting Items for Systematic Reviews and Meta-Analysis (PRISMA) statement (Moher et al., 2009).

\subsection{Literature search}

The following seven online databases were searched on internet by 1 March, 2020 including: Cochrane Library, PubMed, EMBASE, China National Knowledge Infrastructure Database (CNKI), China Biology Medicine disc (CBMdisc), VIP Chinese Science and Technology Periodical Database (VIP), and Wan Fang Database. The following search terms were used individually or combined: "Traditional Chinese medicine" OR "Chinese medicinal" OR "Chinese herb" OR "Chinese herbal medicine" OR "Chinese patent medicine" OR "Chinese medicine preparation") AND ("food therapy" OR "diet therapy" OR "medicated diet" OR "medicinal food" OR "medicinal diet" OR "dietary therapy" AND ("cancer" OR "neoplasm" OR "tumor" OR "carcinoma" OR "malignancy") AND "random". These terms were used as Mesh and free text terms (translated into Chinese) to search the Chinese databases. All relevant publications, including dissertation and conference papers, were researched to ensure a comprehensive search.

\subsection{Inclusion and exclusion criteria}

Inclusion criteria: (1) Only randomized controlled trials (RCTs) were included, regardless of blinding and language. (2) There were no limitations on the patient's gender, age, and the course and severity of the disease. Patients were diagnosed with cancer by pathology or cytology and had accepted chemotherapy and/or radiotherapy. Inclusion and exclusion criteria. (3) The experimental group was treated with TCM diet, and the control group was treated with ordinary diet. Both groups received the same routine treatment.

Exclusion criteria. Animal experiments; duplicate publications and studies involving different evaluation criteria (which means that the outcomes cannot be properly assessed), or presenting incomplete data.

The following outcomes were assessed: (1) Drug related adverse events included leukopenia, hemoglobin, nausea and vomiting. Incidence rate $(\%)=\mathrm{N}$ (number of occurrences) $/ \mathrm{N}$ (total number of cases) $\times 100 \%$. (2) Karnofsky Performance Status (KPS), an indicator for measuring quality of life. The standard (People's Republic of China, 1991) was as follows: Improvement: KPS increased by $\geq 10$ points after treatment; Stable: KPS increased or decreased $<10$ points; Decreased: KPS decreased $>10$ points. (3) Weight: Increased: weight increased by $\geq 1 \mathrm{~kg}$ after treatment; Stable: weight increased or decreased $<1 \mathrm{~kg}$; Decreased: weight decreased $>1 \mathrm{~kg}$.

\subsection{Data extraction and quality assessment}

Two researchers (XZ and LS) independently searched the literature and screened the output. Any difference regarding eligibility during the process were resolved by third-party consultation. GW and MF then independently extracted the data from the studies. The data extracted included: basic study characteristics, sample size, research design, intervention measures, indicators and measurements after the intervention. After extraction, another two researchers (LL and QS) checked the extracted content for accuracy, and in case of any disagreements arising, the two other researchers (LX and YW) would resolve the problem by re-searching the literature and discussing the findings.

\subsection{Risk of bias assessment}

The Cochrane risk of bias assessment tool was used for the assessment (Deng et al., 2016). Two researchers (GW and QS) independently assessed the risk of bias for the included studies. Disagreements were resolved by discussion and consensus, or consultation with a third-party author (L.L).

\subsection{Statistical analysis}

Review Manager Software (RevMan, Version 5.3 for windows, (Cochrane Community, London, United Kingdom) was used to analyze and synthesize the outcomes. Clinical heterogeneity is the primary source of heterogeneity in the systematic reviews of TCMs diet therapy. It can be derived from the potential factors such as the ingredients and formulations of TCMs (Hu et al., 2010). Quantitative synthesis would be done if clinical heterogeneity is not considered by at least two authors in the discussion. Continuous variable was described by mean difference (MD), $\mathrm{P}$ value, and $95 \%$ confidence interval (CI). For dichotomous outcomes, we used the relative risk (RR), with $95 \% \mathrm{CI}$ and $\mathrm{p}$ values, to evaluate the efficacy of TCMs. $\mathrm{I}^{2}$ test was used to judge the heterogeneity of meta-analysis. $\mathrm{I}^{2}$ value $>50 \%$ was considered as an indication of substantial heterogeneity. If heterogeneity existed in the pooled studies, the data were analyzed using a random effects model. If there is significant clinical heterogeneity, the cause of heterogeneity would be explored, and sensitivity analysis or subgroup analysis would be carried out when necessary.

\section{Results}

\subsection{Study characteristics}

In this study, a total of 286 studies was retrieved after searching 7 databases. During the first screening process, 98 related studies were collected, and after reviewing the titles and abstracts, 63 studies were excluded. After reviewing the full texts of 35 studies, 19 studies that did not meet the inclusion criteria were excluded, and the remaining 16 studies ${ }^{10-23,28,29}$ were included in this meta-analysis. The selection process is shown in Figure 1. The basic characteristics of the included studies are detailed in Table 1. The herbal ingredients are shown in Attachment data 1. 
The risk of bias for each study was assessed according to Section 5.1.0 of the Cochrane Handbook for Systematic Reviews of Interventions. The outcome reporting was found to be completely consistent in the results section and methodology section. The results of the risk of bias assessment are shown in Figures $2 \mathrm{~A}$ and $\mathrm{B}$ ).

\subsection{Outcomes}

Leukopenia

Table 2 depicts the changes in leukopenia attenuating levels. All 6 trials (Chang et al., 2011; Huang et al., 2011; Wang et al., 2011, 2012; Shi et al., 2012; Zhang, 2009) ( $n=590)$ reported significantly
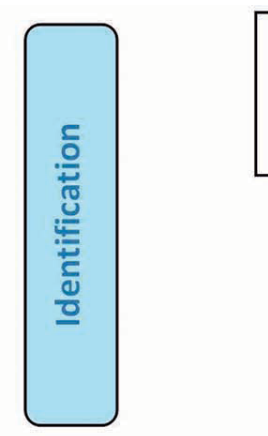

$$
\begin{aligned}
& \text { records identified through } \\
& \text { database searching(n=286) }
\end{aligned}
$$
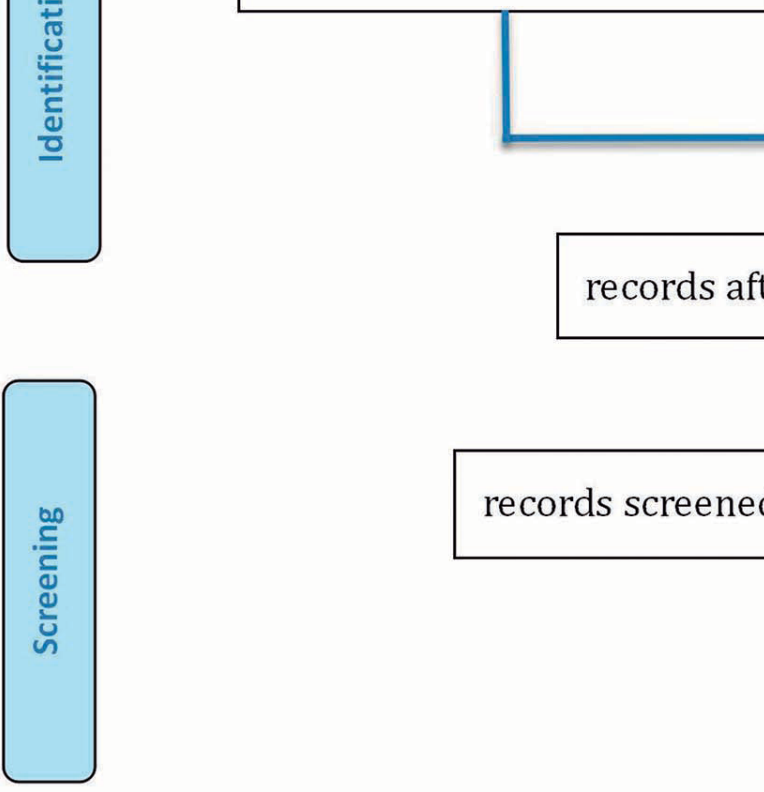

$$
\begin{aligned}
& \text { additional records identified } \\
& \text { through other sources(n=0) }
\end{aligned}
$$

ecords after duplicates removed(n=98)
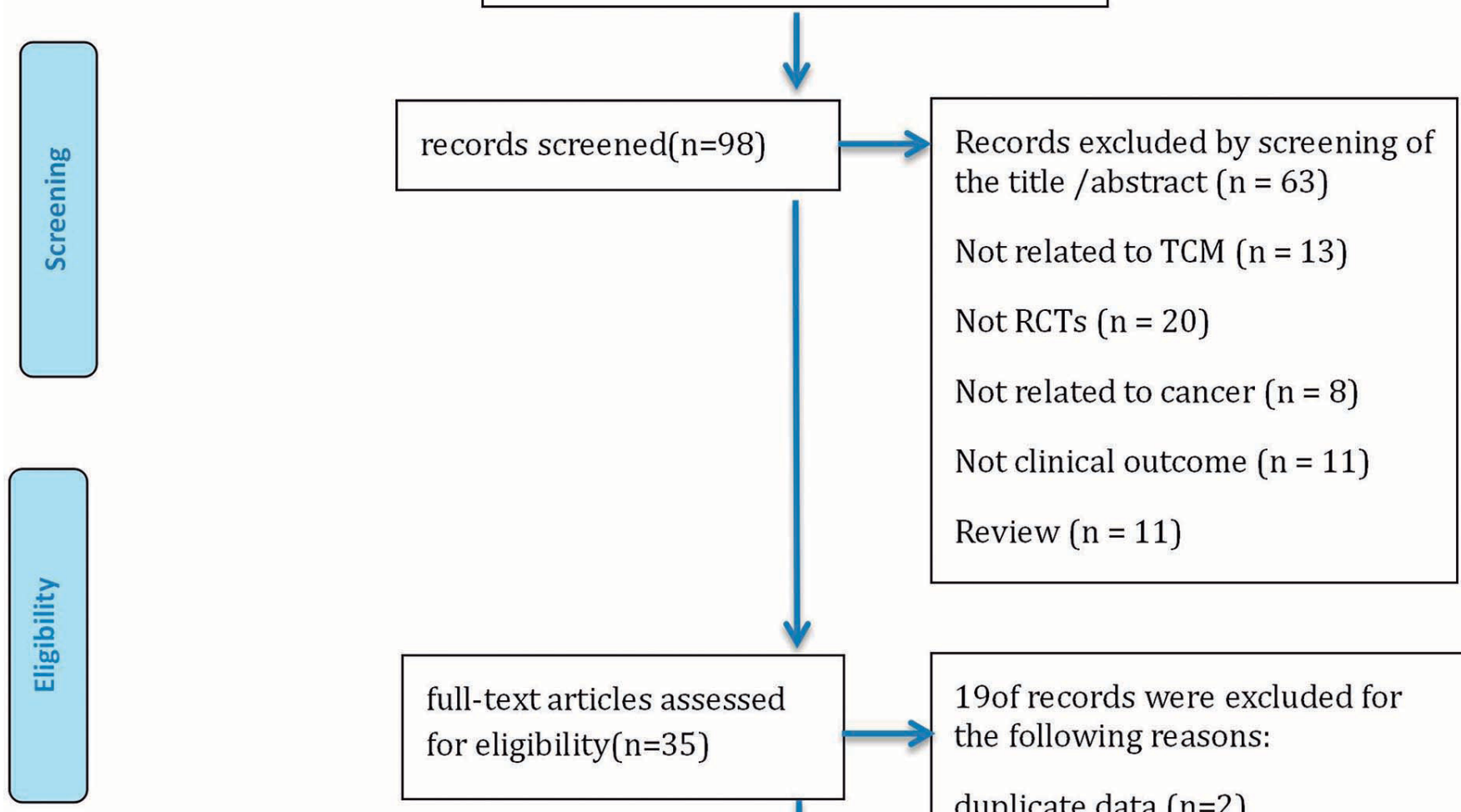

studies included in qualitative synthesis(n=16)
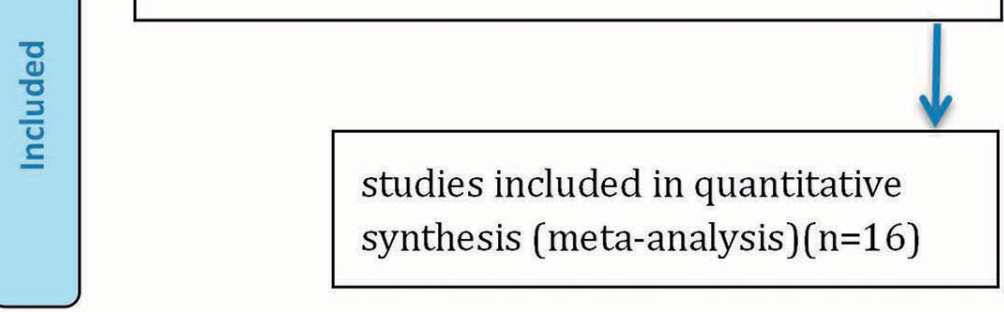

19of records were excluded for the following reasons:

duplicate data $(\mathrm{n}=2)$

not TCM diet therapy $(\mathrm{n}=2)$

not $\operatorname{RCT}(n=6)$

inconsistency with outcomes $(n=2)$

No relative outcomes $(n=7)$

Figure 1. Selection process of meta-analysis. 
Table 1. Characteristic of included studies.

\begin{tabular}{|c|c|c|c|c|c|c|c|}
\hline \multirow{2}{*}{$\begin{array}{l}\text { Study (author, } \\
\text { year) }\end{array}$} & \multirow{2}{*}{$\begin{array}{c}\text { Sample } \\
\text { size }\end{array}$} & \multicolumn{2}{|c|}{ Age (years) } & \multicolumn{2}{|c|}{$\begin{array}{c}\text { Sex (cases) } \\
\text { (Male/Female) }\end{array}$} & \multirow[t]{2}{*}{ Intervention and control protocol } & \multirow[t]{2}{*}{ Outcomes } \\
\hline & & C & E & $\mathrm{C}$ & $\mathrm{E}$ & & \\
\hline $\begin{array}{l}\text { Chang et al. } \\
\text { (2011) }\end{array}$ & $46 / 46$ & $46-75(62.1)$ & $45-73(61.2)$ & $16 / 44$ & $14 / 46$ & $\begin{array}{l}\text { C: RT + ordinary therapy and diet; E: RT + } \\
\text { Invigorating Spleen and Replenishing Qi, } \\
\text { Strengthening Kidney and Nourishing Yin } \\
\text { Combined with TCM diet. }\end{array}$ & (1) (2) (4) \\
\hline $\begin{array}{l}\text { Huang et al. } \\
\text { (2011) }\end{array}$ & $63 / 63$ & $47-76(62.4)$ & $46-78(62.3)$ & $35 / 28$ & $34 / 29$ & $\begin{array}{l}\text { C: RT + ordinary therapy and diet; E: RT + herbs } \\
\text { (Benefiting qi and nourishing yin, Strengthening } \\
\text { Spleen and harmonize the stomach,solid kidney, } \\
\text { Detoxication and dissolve yu) + TCM diet therapy. }\end{array}$ & (2) (4) \\
\hline $\begin{array}{l}\text { Wang et al. } \\
\text { (2011) }\end{array}$ & $66 / 66$ & $45-78(60.5)$ & $47-77(61.8)$ & $36 / 30$ & $37 / 29$ & $\begin{array}{l}\text { C: CH and RT + ordinary therapy and diet; E: } \\
\text { CH and RT + Righting Thanh Hoa Chinese } \\
\text { soup(bulk) + TCM diet Righting Thanh Hoa } \\
\text { Chinese soup(bulk): qd when stage of therapy. } \\
\text { Yin blood deficiency: Baiyin Yizao drink, } \\
\text { Heimuer congee, Shouwu Jiyu Decoction. Yang } \\
\text { qi deficiency: Guiyuan Shanyao congee, Renshen } \\
\text { Lentinula Decoction, Huangqi chicken soup. }\end{array}$ & (2) (4) \\
\hline $\begin{array}{l}\text { Wang et al. } \\
\text { (2012) }\end{array}$ & $58 / 58$ & $45-73(62.38 \pm 6.75)$ & $43-74(61.66 \pm 7.25)$ & $14 / 44$ & $13 / 45$ & $\begin{array}{l}\text { C: CH and RT + ordinary therapy and diet; E: CH and } \\
\text { RT + Fuzheng Peiben Qinghua Decoction+ TCM diet } \\
\text { Yang qi deficiency: Guiyuan Shanyao congee, Renshen } \\
\text { Lentinula Decoction, Huangqi chicken soup. }\end{array}$ & (1)(2)(3) (4) \\
\hline $\begin{array}{l}\text { Shi et al. } \\
\text { (2012) }\end{array}$ & 36 & & & & & $\begin{array}{l}\text { C: } \mathrm{CH}+\text { ordinary therapy and diet; E: } \mathrm{CH}+\mathrm{TCM} \\
\text { diet (Wuhong Decoction: Fructus Lycii, Jujube, } \\
\text { Soft legume ormosia seed, Peanuts, Brown sugar) }\end{array}$ & (2) \\
\hline Zhang (2009) & $30 / 30$ & 46 & 46 & - & - & $\begin{array}{l}\mathrm{C}: \mathrm{CH}+\text { ordinary therapy and diet; } \mathrm{E}: \mathrm{CH}+\text { the food } \\
\text { of Strengthening Spleen nourishing stomach, and } \\
\text { Removing Dampness detoxication (Baihe stewed } \\
\text { pork tripe, Lemon juice, Huanghua stewed fish) }\end{array}$ & (1) (2) \\
\hline Ao (2013) & $22 / 18$ & $40-72(61)$ & $40-72(61)$ & $10 / 18$ & $12 / 10$ & $\begin{array}{l}\mathrm{C}: \mathrm{CH} \text { +ordinary therapy and diet; E: } \mathrm{CH} \text { +ordinary } \\
\text { therapy+ TCM diet therapy (TCM food: Huangqi } \\
\text { stewed pigeon; Peanut Seed-coat and Jujube juice. } \\
\text { Heimuer and brown sugar drink; Shengui pigeon; } \\
\text { Ejiao milk. TCM congee: Ejiao congee. Shanyao- } \\
\text { biandou-jijin congee; Gouqi Hongzao congee. }\end{array}$ & (2) \\
\hline $\begin{array}{l}\text { Pan \& Chen } \\
(2001)\end{array}$ & $38 / 40$ & $18-74(51.2)$ & $18-74(51.2)$ & & & $\begin{array}{l}\mathrm{C}: \mathrm{CH} \text { and } \mathrm{RT}+\text { ordinary therapy and diet; } \mathrm{E}: \mathrm{CH} \text { and } \\
\mathrm{RT} \text { +ordinary therapy+Zibu congee, Shenzao rice,. }\end{array}$ & (2) \\
\hline $\begin{array}{l}\text { Yue et al. } \\
\text { (2007) }\end{array}$ & $84 / 84$ & & & $52 / 32$ & $51 / 33$ & $\begin{array}{l}\mathrm{C}: \mathrm{CH}+\text { ordinary therapy and diet; } \mathrm{E}: \mathrm{CH} \\
\text { +ordinary therapy }+\mathrm{TCM} \text { diet therapy (Dihuang } \\
\text { congee; Lianrou oral thick paste). }\end{array}$ & (1) (2) (4) \\
\hline $\begin{array}{l}\text { Zhang et al. } \\
\text { (2014) }\end{array}$ & $40 / 38$ & $50.8 \pm 9.8$ & $51.8 \pm 8.3$ & $30 / 8$ & $28 / 12$ & $\begin{array}{l}\text { C: } \mathrm{CH} \text { and(or) RT +ordinary therapy and diet; E: } \\
\mathrm{CH} \text { and(or) RT + ordinary therapy + the food of } \\
\text { Strengthening Spleen nourishing stomach, and } \\
\text { Removing Dampness detoxication. }\end{array}$ & (1) (2) \\
\hline $\begin{array}{l}\text { Liu et al. } \\
\text { (2015) }\end{array}$ & $94 / 94$ & & & & & $\begin{array}{l}\mathrm{C}: \mathrm{CH}+\text { ordinary therapy and diet; } \mathrm{E}: \mathrm{CH} \\
\text { +ordinary therapy+TCM diet therapy (Dihuang } \\
\text { congee, Lianrou oral thick paste). }\end{array}$ & (1) (2) (4) \\
\hline $\begin{array}{l}\text { Fang et al. } \\
(2018)\end{array}$ & $30 / 30$ & $42-68(55)$ & $42-68(55)$ & & 128 & $\begin{array}{l}\mathrm{C}: \mathrm{CH}+\text { ordinary therapy and diet; } \mathrm{E}: \mathrm{CH}+ \\
\text { ordinary therapy+ Lizhong decoction and } \\
\text { Zhulimilk juice. }\end{array}$ & (1) (3) \\
\hline Qu (2018) & $38 / 38$ & $23-72(54.51 \pm 0.63)$ & $22-71(54.12 \pm 0.63)$ & $20 / 18$ & $21 / 17$ & $\begin{array}{l}\text { C: } \mathrm{CH} \text { +ordinary therapy and diet; E: } \mathrm{CH} \\
\text { +ordinary therapy+TCM diet therapy TCM diet } \\
\text { (Qi and blood tonification congee; Colla Corii } \\
\text { Asini and peanut congee). }\end{array}$ & (1) \\
\hline $\begin{array}{l}\text { Jiang et al. } \\
\text { (2015) }\end{array}$ & $32 / 32$ & $35-74(55.8 \pm 16.2)$ & $38-76(56.2 \pm 15.7)$ & $16 / 16$ & $15 / 17$ & $\begin{array}{l}\mathrm{C}: \mathrm{CH} \text { and RT + ordinary therapy and diet; E: } \\
\mathrm{CH} \text { and RT + ordinary therapy+ TCM diet (Qi } \\
\text { and blood tonification congee; Ejiao and peanut } \\
\text { congee; Guiyuan Dazao congee). }\end{array}$ & (1)(3) (4) \\
\hline Wu (2016) & $35 / 35$ & $37-75(55.4 \pm 16.1)$ & $39-77(56.9 \pm 17.5)$ & $19 / 16$ & $17 / 18$ & $\begin{array}{l}\text { C: } \mathrm{CH} \text { and } \mathrm{RT}+\text { ordinary therapy and diet; E: } \mathrm{CH} \\
\text { and RT +ordinary therapy+ TCM diet (Qi and } \\
\text { blood tonification congee). }\end{array}$ & (3) (4) \\
\hline $\begin{array}{l}\text { Li \& Zhu } \\
(2012)\end{array}$ & $43 / 43$ & $37-71(54.5)$ & $36-69(52.5)$ & & & $\begin{array}{l}\mathrm{C}: \mathrm{CH}+\text { ordinary therapy and diet; } \mathrm{E}: \mathrm{CH}+ \\
\text { ordinary therapy + Shenqi Jianpi Decoction. }\end{array}$ & (3) \\
\hline
\end{tabular}

C: control group; E: experiment group; RT: radiotherapy; CH: chemotherapy; (1) Gastrointestinal reaction; (2)bone marrow depression; (3): KPS score; (4)Weight. 


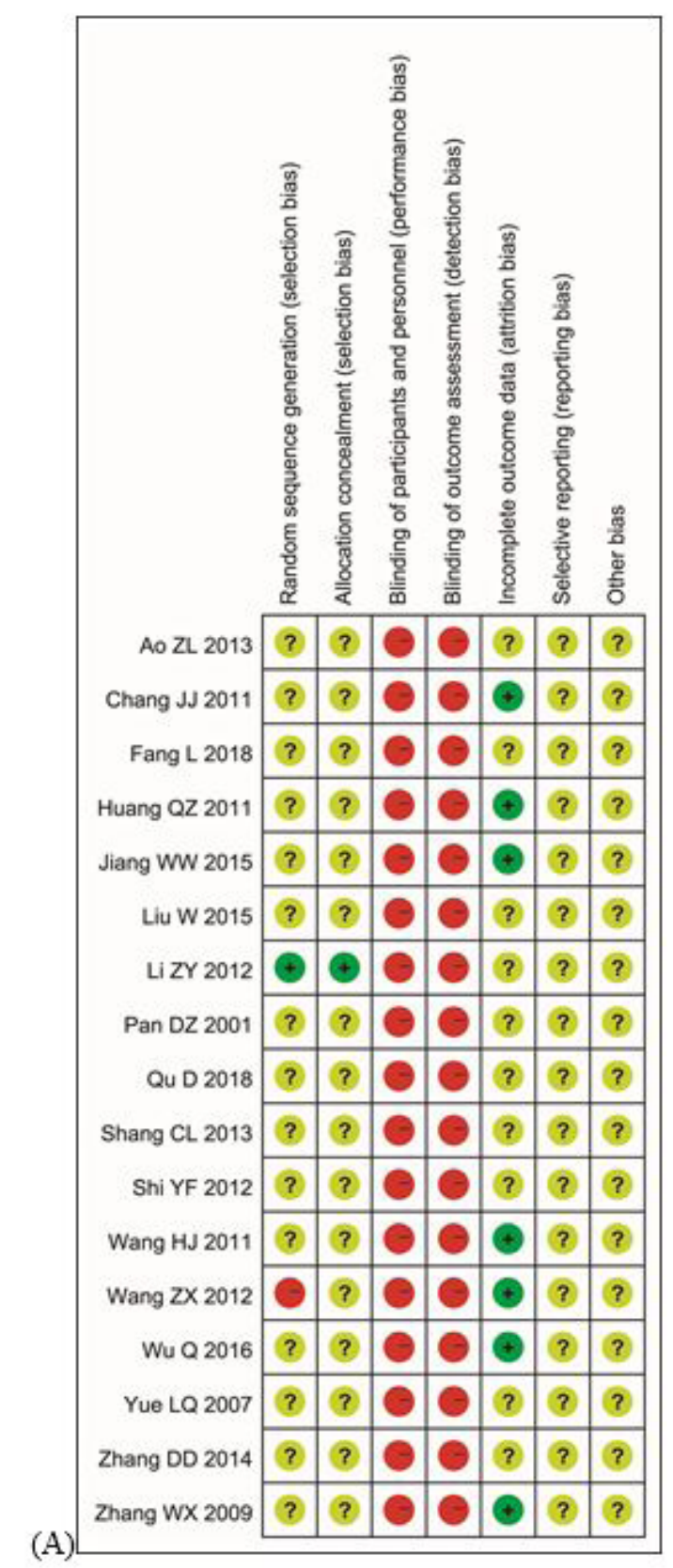

(B)

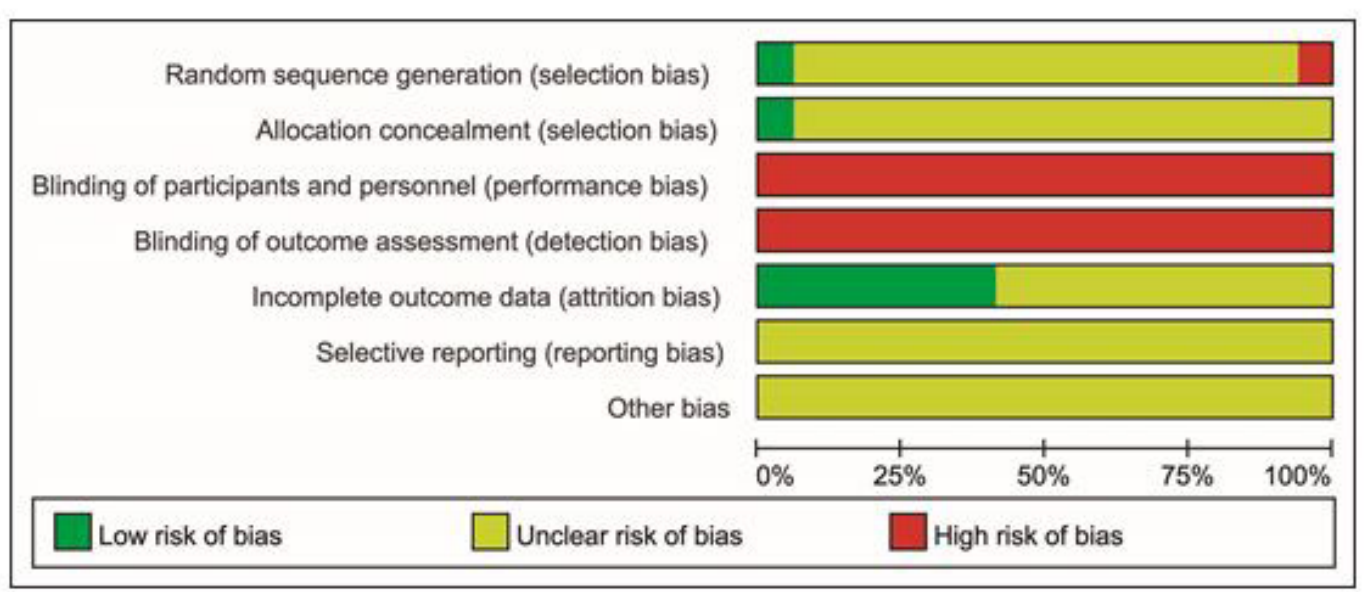

Figure 2. Risk of bias assessment (A) Summary (B) Graph. 
changed in leukopenia attenuating levels in the treatment groups in contrast to the control groups. All leukopenia was relatively homogenous through trials, allowing for a fixed-effects model to be employed for statistical analysis $\left(\mathrm{P}=0.49, \mathrm{I}^{2}=0 \%\right)$. The impact of TCM diet on leukopenia was significantly different $[\mathrm{RR}=0.57 ; 95 \%$ $\mathrm{CI}(0.47,0.69) ; \mathrm{P}<0.0001]$, compared with patients using ordinary diet. Four trials making comparison of TCM diet + Chinese herbs with ordinary diet, and TCM diet + Chinese herbs was proved to be superior to ordinary diet $[\mathrm{RR}=0.58 ; 95 \% \mathrm{CI}(0.46,0.72)$; $\mathrm{P}<0.0001]$. Two trials compared TCM diet therapy with ordinary diet, the result shows that TCM diet was superior to ordinary $\operatorname{diet}[\mathrm{RR}=0.56 ; 95 \% \mathrm{CI}(0.38,0.81) ; \mathrm{P}<0.0001]$, and there was no significantly different between the two subgroups $\left(\mathrm{P}=0.86, \mathrm{I}^{2}=0 \%\right)$.

\section{Hemoglobin}

Five studies (Ao, 2013; Pan \& Chen, 2001; Yue et al., 2007; Zhang et al., 2014; Liu et al., 2015) including 540 cases reported the hemoglobin level. Heterogeneity analysis showed that 5 studies had statistical homogeneity $\left(\mathrm{P}=0.69, \mathrm{I}^{2}=0 \%\right)$. As shown in Table 3, the meta-analysis revealed that hemoglobin in the experimental group was statistically higher than that in the control group $[\mathrm{MD}=10.04 ; 95 \% \mathrm{CI}(7.66,12.41) ; \mathrm{P}<0.0001]$.

\section{Nausea and vomiting}

Nine trials(Chang et al., 2011; Wang et al., 2011; Zhang, 2009; Yue et al., 2007; Zhang et al., 2014; Liu et al., 2015; Fang et al., 2018; Qu, 2018; Jiang et al., 2015) including 930 participants reported TCM diet therapy for alleviation symptoms of nausea and vomiting. Random-effects model was used with no heterogeneity $\left(\mathrm{P}=0.004, \mathrm{I}^{2}=65 \%\right)$, and our pooled results showed that TCM diet therapy could further increase the body weight compared with the control group $[R R=0.54,95 \% C I=(0.43,0.70)$, $\mathrm{P}=0.004]$. Four studies compared the occurrence rates of TCM diet + Chinese herbs with ordinary diet as a subgroup, and TCM diet + Chinese herbs was proved to be more efficient in relieving the symptoms of nausea and vomiting $[\mathrm{RR}=0.66,95 \% \mathrm{CI}(0.49$, $0.91), \mathrm{P}=0.01]$. Five trials making comparison of TCM diet and ordinary diet, and TCM diet was suggested to be more effective in symptoms relief [RR, 0.47, 95\% CI, $(0.37,0.60), \mathrm{P}<0.0001]$. Furthermore, the differences in the incidence rate of nausea and vomiting were not statistically significant based on subgroup analysis $\left(\mathrm{P}=0.09, \mathrm{I}^{2}=66.2 \%\right)$ ] (Figure 3 ). In Figure 4, the funnel plots for comparison of the incidence of nausea and vomiting was roughly symmetrical, indicating low risk of publication bias.

\section{KPS}

Figure 5 depicts the change in KPS improvement rates. All 5 trials (Wang et al., 2011; Fang et al., 2018; Jiang et al., 2015; Wu, 2016; Li \& Zhu, 2012) $(n=396)$ reported significantly changed in KPS improvement rates in the treatment groups in contrast to the control groups. All KPS improvement rates were relatively homogeneous through trials, allowing for a fixed-effects model to be employed for statistical analysis. The impact of TCM diet therapy on KPS improvement rates was significantly higher $[\mathrm{RR}=1.28$; $95 \% \mathrm{CI}$ $(1.15,1.42) ; \mathrm{P}<0.0001]$, compared with patients using ordinary diet. Two trials making comparison of TCM diet + Chinese herbs with ordinary diet, and TCM diet + Chinese herbs was suggested to be superior to ordinary diet $[\mathrm{RR}=1.29 ; 95 \% \mathrm{CI}(1.07,1.56) ; \mathrm{P}=0.009]$. Three trials compared TCM diet therapy with ordinary diet, the result shows that TCM diet was superior to ordinary diet $[\mathrm{RR}=1.27 ; 95 \%$ $\mathrm{CI}(1.13,1.43) ; \mathrm{P}<0.0001]$, and there was no significantly differences between the two subgroups $\left(\mathrm{P}=0.88, \mathrm{I}^{2}=0 \%\right)$.

\section{Weight}

Eight RCTs (Chang et al., 2011; Huang et al., 2011; Wang et al., 2011, 2012; Yue et al., 2007; Liu et al., 2015;

Table 2. Analysis of leukopenia of TCM diet combined with CH (and/or RT) (treatment) versus ordinary diet (control).

\begin{tabular}{|c|c|c|c|c|c|c|}
\hline & Subgroup & $\begin{array}{c}\text { No. of participant } \\
\text { (studies) }\end{array}$ & $95 \% \mathrm{CI}$ & $\mathrm{Z}$ & $\mathrm{P}$ & Subgroup difference \\
\hline \multirow[t]{2}{*}{ Leukopenia } & $\begin{array}{c}\text { TCM diet+Chinese } \\
\text { herbs }\end{array}$ & 4 & $0.58[0.46,0.72]$ & 4.94 & $\mathrm{P}<0.0001$ & \multirow[t]{3}{*}{$\mathrm{P}=0.86$} \\
\hline & TCM diet & 2 & $0.56[0.38,0.81]$ & 3.09 & $\mathrm{P}=0.002$ & \\
\hline Total $(95 \% \mathrm{CI})$ & & & $0.57[0.47,0.69]$ & & & \\
\hline
\end{tabular}

TCM: Traditional Chinese medicine; RT: radiotherapy; CH: chemotherapy. P: Probability. Z: Zhi. 95\%CI: confidence interval.

Table 3. Analysis of hemoglobin of TCM diet combined with CH (and/or RT) (treatment) versus ordinary diet (control).

\begin{tabular}{|c|c|c|c|c|c|c|c|c|}
\hline Study (author, year) & \multicolumn{3}{|c|}{ Experimental } & \multicolumn{3}{|c|}{ Control } & \multirow{2}{*}{ Weight } & \multirow{2}{*}{ Mean Difference } \\
\hline Group & Mean & $\mathrm{SD}$ & Total & Mean & $\mathrm{SD}$ & Total & & \\
\hline Ao $(2013)$ & 105.73 & 5.84 & 22 & 95.2 & 4.79 & 18 & $52.00 \%$ & $10.53[7.24,13.82]$ \\
\hline Liu et al. (2015) & 113 & 20 & 94 & 103 & 28 & 94 & $11.70 \%$ & $10.00[3.04,16.96]$ \\
\hline Pan \& Chen (2001) & 106 & 14.6 & 40 & 94.1 & 16.5 & 26 & $9.30 \%$ & $11.90[4.11,19.69]$ \\
\hline Yue et al. (2007) & 114 & 21 & 84 & 102 & 27 & 84 & $10.50 \%$ & $12.00[4.69,19.31]$ \\
\hline Zhang et al. (2014) & 141.9 & 12.7 & 40 & 135.7 & 13.6 & 38 & $16.50 \%$ & $6.20[0.35,12.05]$ \\
\hline Total (95\% CI) & & & 280 & & & 260 & $100.00 \%$ & $10.04[7.66,12.41]$ \\
\hline
\end{tabular}

Heterogeneity: $\mathrm{Chi}^{2}=2.24, \mathrm{df}=4(\mathrm{P}=0.69) ; \mathrm{I}^{2}=0 \% . \mathrm{SD}$ : standard deviation

Test for overall effect: $\mathrm{Z}=8.28(\mathrm{P}<0.00001)$

TCM: Traditional Chinese medicine; RT: radiotherapy; $\mathrm{CH}$ : chemotherapy. 
Zhou et al.

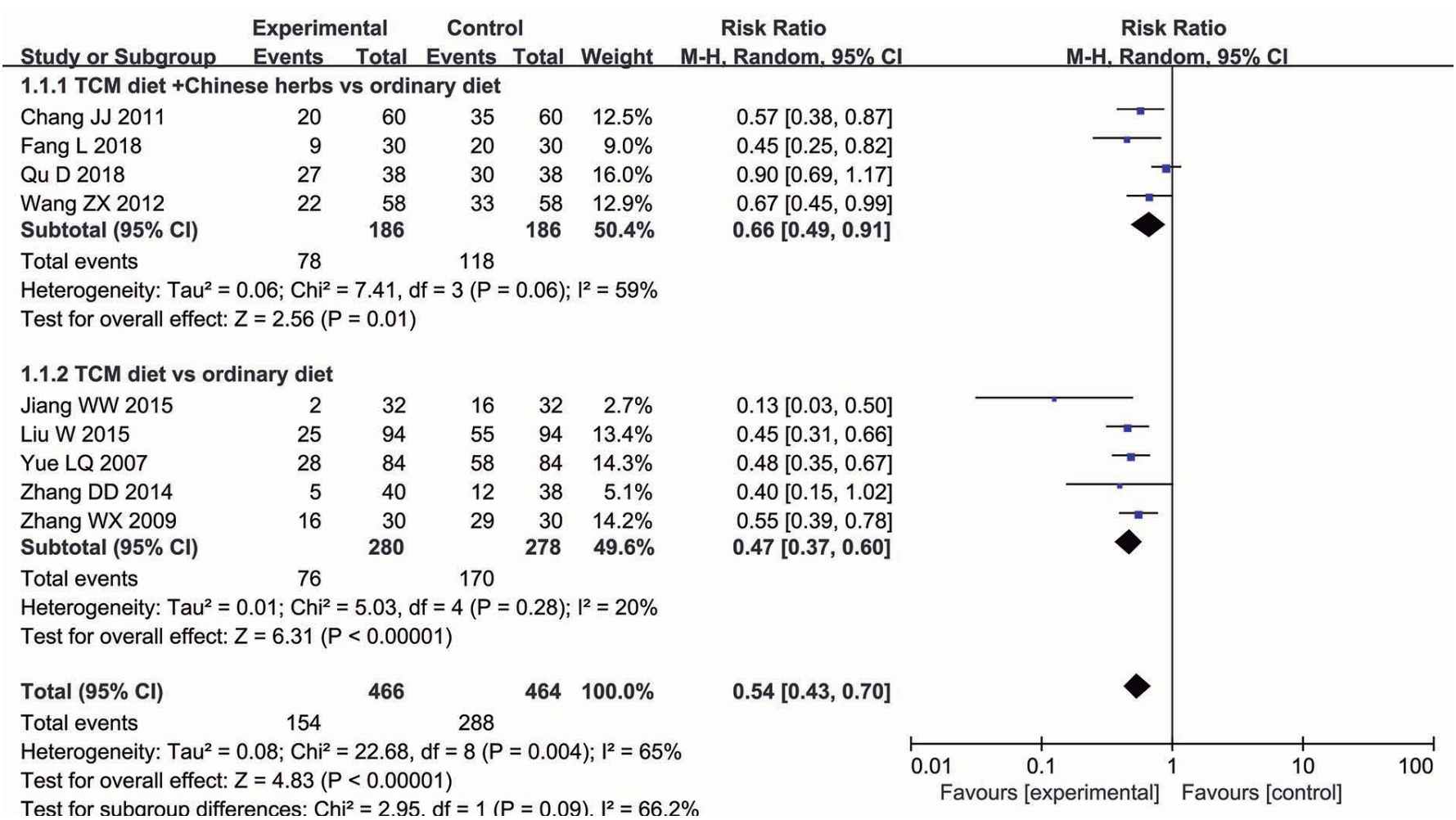

Figure 3. Subgroup analysis on the incidence rate of nausea and vomiting.

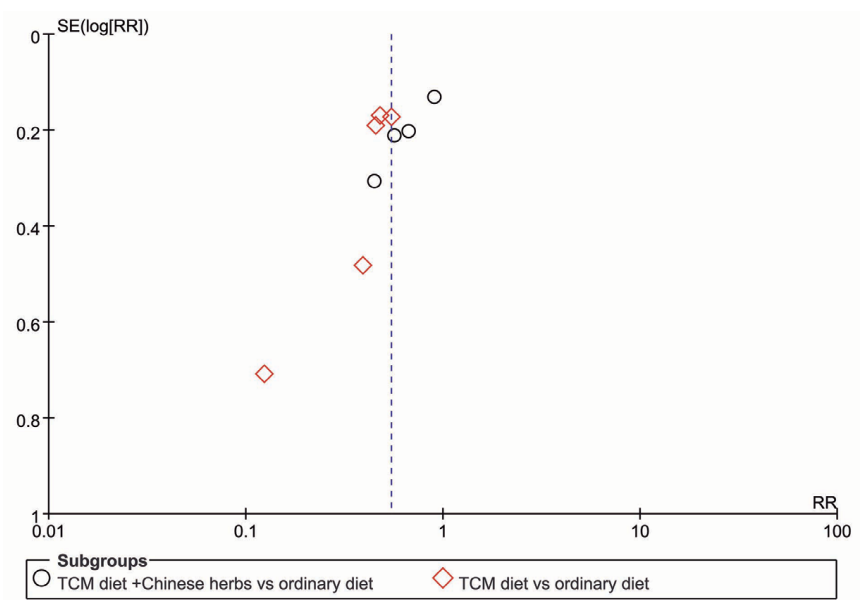

Figure 4. Funnel plots for comparison of the incidence of nausea and vomiting. SE: standard error; RR: relative risk.

Jiang et al., 2015; Wu, 2016) reported the change of weight. Four studies (Chang et al., 2011; Huang et al., 2011; Wang et al., 2011, 2012) compared TCM diet + Chinese herbs with ordinary diet, and four studies (Yue et al., 2007; Liu et al., 2015; Jiang et al., 2015; Wu, 2016) compared TCM diet therapy with ordinary diet. Randomeffects model was used with no heterogeneity $\left(\mathrm{P}=0.65, \mathrm{I}^{2}=0 \%\right)$, and our pooled results showed that TCM diet therapy could further increase the body weight compared with control group $[R R=1.53,95 \% C I=(1.38,1.68), \mathrm{P}<0.0001]$. Subgroup analysis also showed that TCM diet + Chinese herbs was significantly better than ordinary diet $[\mathrm{RR}=1.59,95 \% \mathrm{CI}=(1.33,1.90)$, $\mathrm{P}<0.0001]$. Four trials making comparison between TCM diet
+ Chinese herbs and ordinary diet, and TCM diet + Chinese herbs was proved to be superior to ordinary diet $[R R=1.59$; $95 \% \mathrm{CI}(1.33,1.90) ; \mathrm{P}<0.0001]$. Four trials compared TCM diet therapy with ordinary diet, and the result shows that TCM diet was superior to ordinary diet $[\mathrm{RR}=1.50,95 \% \mathrm{CI}=(1.33,1.69)$; $\mathrm{P}<0.0001]$, and there was no significantly difference between the two subgroups $\left(\mathrm{P}=0.61, \mathrm{I}^{2}=0 \%\right)$ (Table 4$)$.

\section{Discussion}

This meta-analysis of sixteen RCTs including 2336 cases comparing the efficacy of TCM diet with ordinary diet. Our results suggested that TCM diet therapy is superior to ordinary diet in terms of reducing the incidence of leukopenia, and nausea and vomiting, increasing hemoglobin, improving KPS, and increasing weight in patients with cancer undergoing chemotherapy (and/or radiotherapy). Based on the data of KPS improvement (Wang et al., 2011; Fang et al., 2018; Jiang et al., 2015; Wu, 2016; Li \& Zhu, 2012) and weight gain (Chang et al., 2011; Huang et al., 2011; Wang et al., 2011, 2012; Yue et al., 2007; Liu et al., 2015; Jiang et al., 2015; Wu, 2016) TCM diet may improve the quality of life for cancer patients receiving chemotherapy (and/or radiotherapy). Some of the included trials (Chang et al., 2011; Huang et al., 2011; Wang et al., 2011, 2012) reported that it was beneficial for tumor response and prolonged survival rate, but they need to be further studied with larger sample size and non-single centered studies. As far as we know, this is the first registered neta-analysis that evaluate the effects of TCM diet therapy in alleviating the toxicity in cancer patients undergoing chemotherapy (and/or radiotherapy) receiving diet guides. 


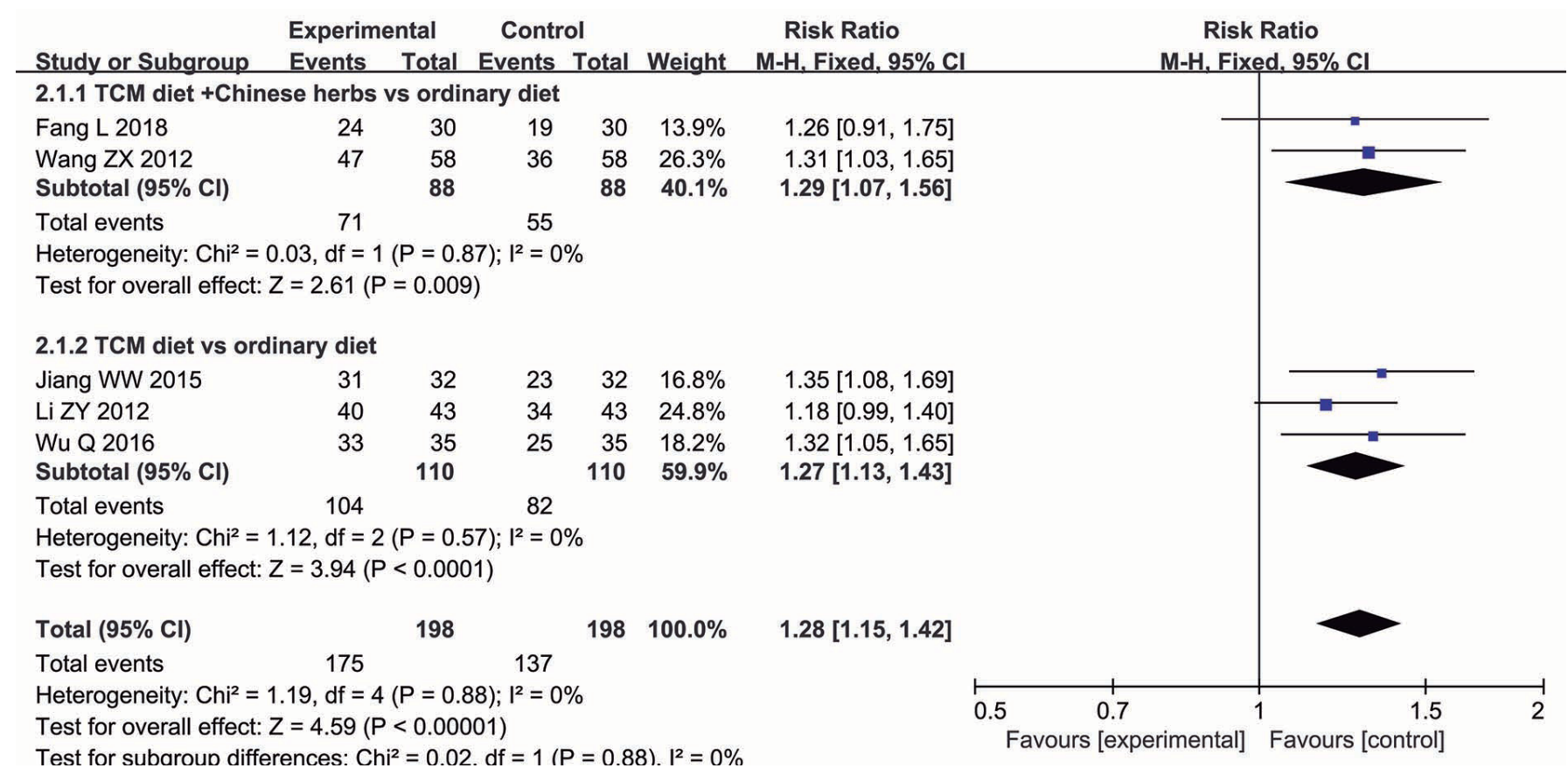

Figure 5. Subgroup analysis on the change in KPS improvement rates.

Table 4. Analysis of body weight changes in TCM diet combined with CH (and/or RT) (treatment) versus ordinary diet (control).

\begin{tabular}{lcccc}
\hline \multicolumn{1}{c}{ Subgroup } & No. of participant (studies) & $95 \% \mathrm{CI}$ & $\mathrm{Z}$ & $\mathrm{P}$ \\
\hline TCM diet+Chinese herbs & 4 & $1.59[1.33,1.90]$ & 5.06 & $\mathrm{P}<0.00001$ \\
TCM diet & 4 & $1.50[1.33,1.69]$ & 6.77 & $\mathrm{P}<0.00001$ \\
subgroup difference & & & $\mathrm{P}=0.61$ \\
Total (95\% CI) & & $1.53[1.38,1.68]$ & \\
\hline
\end{tabular}

TCM: Traditional Chinese medicine; RT: radiotherapy; $\mathrm{CH}$ : chemotherapy.

Our data suggest that TCM diet can play a more comprehensive role as a therapy for alleviating side effects in patients undergoing chemotherapy (and/or radiotherapy). A prospective, non-randomized longitudinal study (Liew et al., 2019) indicated that Chinese herbs could reduce fatigue, nausea, and anorexia in breast cancer patients, but did not decrease chemotherapy-associated hematologic toxicity. It might due to the reason that it was a non-randomized controlled study, and too few cases, which may have an impact on the results. Another study (Zheng et al., 2017) investigated the relationship between Chinese medicine dietary patterns (hot, neutral, and cold) and the incidence of breast cancer among Chinese women in Hong Kong. It indicated that non-breast cancer women adopted a neutral dietary pattern, and chose Chinese medicine supplements more frequently.

However this study is not without limitations. First, the included trials were all Chinese though we searched through 7 databases. Further researches are needed to evaluate the efficacy of TCM diet in other populations. Second, the methodological quality of the included studies should be improved. Though most trials performed randomization, only a few studies adopted an appropriate random grouping method. Moreover, all trials were carried out at a single centre. Even if these limitations may reduce the effectiveness of this systematic review, the included studies were rigorously screened and considered to be highly comparable. These results indicate that further research on Chinese medicine diet in the treatment of cancer is necessary. In order to conclude the efficacy of TCM diet, large-scale randomized multicenter clinical trials with long-term follow up are required.

In general, the findings of this meta-analysis demonstrated that TCM diet may reduce the toxicity of chemotherapy (and/or radiotherapy). However, further more double-blind, multicenter, large-scale RCTs, high-quality RCTs of standardized design and conducted according to the principles of evidence-based medicine are needed. Our results suggested that TCM diet therapy is superior to ordinary diet in terms of reducing incidence of leukopenia, and nausea and vomiting during chemotherapy (radiotherapy), improving KPS, increasing hemoglobin, gaining weight in patients with cancer undergoing chemotherapy and(or) radiotherapy.

\section{Acknowledgements}

This work is supported by the Medical and Health Technology Foundation of Zhejiang Project, Project number: 2019ZH048; Technology Foundation of Tongxiang Project, Project number: 201801013. 


\section{References}

Ao, Z. L. (2013). Therapeutic effect of diet therapy on anemia after chemotherapy in advanced non-small cell lung cancer. Chinese General Practice Nursing, 11, 2351-2352.

Bray, F., Ferlay, J., Soerjomataram, I., Siegel, R. L., Torre, L. A., \& Jemal, A. (2018). Global cancer statistics 2018: GLOBOCAN estimates of incidence and mortality worldwide for 36 cancers in 185 countries. CA: a Cancer Journal for Clinicians, 68(6), 394-424. http://dx.doi. org/10.3322/caac.21492. PMid:30207593.

Chang, J. J., Wang, H. J., Wang, Z. X., Huang, Q. Z., Zhang, P. J., \& Zhang, Z. (2011). Clinical research of therapy of invigorating spleen and replenishing qi, strengthening kidney and nourishing yin combined with medicated diet in treating commonly-encountered middle-late underbelly malignant tumor. Journal of New Chinese Medicine, 43, 90-92.

Crawford, J., Ozer, H., Stoller, R., Johnson, D., Lyman, G., Tabbara, I., Kris, M., Grous, J., Picozzi, V., Rausch, G., Smith, R., Gradishar, W., Yahanda, A., Vincent, M., Stewart, M., \& Glaspy, J. (1991). Reduction by granulocyte colony-stimulating factor of fever and neutropenia induced by chemotherapy in patients with small-cell lung cancer. The New England Journal of Medicine, 325(3), 164-170. http://dx.doi. org/10.1056/NEJM199107183250305. PMid:1711156.

Deng, W. Y., Xu, Y. F., Li, N., \& Luo, S. X. (2016). Observation on clinical effects of Xiaoai ping injection combined with TP regimen in advanced gastric cancer. Chinese Journal of Geriatric Care, 14, 51-52.

Fang, L., Liang, H., Liu, D. M., \& Deng, X. Q. (2018). Decoction combined with dietary therapy for reducing 30 cases of gastrointestinal side effects after chemotherapy of gastric cancer. Chiang-Hsi Chung I Yao, 4, 55-56.

Huang, Q. Z., Wang, H. J., Chang, J. J., Wang, Z. X., Zhang, P. J., \& Zhang, Z. (2011). Clinical research combined with Spleen Qi Gu Shen medicated chest and nourishing yin common treatment of advanced malignant tumor. Zhejiang Journal of Traditional Chinese Medicine, 46, 640-641.

Hsieh, R. K., Chan, A., Kim, H. K., Yu, S., Kim, J. G., Lee, M. A., Dalén, J., Jung, H., Liu, Y. P., Burke, T. A., \& Keefe, D. M. (2015). Baseline patient characteristics, incidence of CINV, and physician perception of CINV incidence following moderately and highly emetogenic chemotherapy in Asia Pacific countries. Supportive Care in Cancer, 23(1), 263-272. http://dx.doi.org/10.1007/s00520-014-2373-2. PMid:25120009.

Hu, D., Kang, D. Y., \& Hong, D. (2010). Heterogeneity analysis of systematic reviews on traditional Chinese medicine. Chinese Journal of Evidence-Based Medicine, 10, 488-491.

Jiang, W. W., Li, L. F., \& Yang, J. W. (2015). Effect of traditional Chinese dietary therapy on rehabilitation of cancer patients: nursing of integrated tradition Chinese and western medicine. Nursing of Integrated Traditional Chinese and Western Medicine, 1, 10-12.

Jordan, K., Gralla, R., Jahn, F., \& Molassiotis, A. (2014). International antiemetic guidelines on chemotherapy induced nausea and vomiting (CINV): content and implementation in daily routine practice. European Journal of Pharmacology, 722, 197-202. http://dx.doi. org/10.1016/j.ejphar.2013.09.073. PMid:24157984.

Li, Q., Liu, J., Lin, H. S., \& Wang, Y. (2017). Diet therapy nutrition recommendation for colorectal cancer patients: based on Chinese theory. Journal of Traditional Chinese Medicine, 20, 1746-1749.

Li, Z. Y., \& Zhu, H. T. (2012). Treatment of advanced ovarian cancer based on dietary supplements of traditional Chinese medicine combined with chemotherapy. Zhongguo Shiyan Fangjixue Zazhi, 18, 332-336.
Liew, A. C., Peh, K. K., Tan, B. S., Zhao, W., \& Tangiisuran, B. (2019). Evaluation of chemotherapy-induced toxicity and health-related quality of life amongst early-stage breast cancer patients receiving Chinese herbal medicine in Malaysia. Supportive Care in Cancer, 27(12), 4515-4524. http://dx.doi.org/10.1007/s00520-019-04724-1. PMid:30911917.

Liu, W., Sun, Y. P., \& Chen, L. (2015). The influence of diet peripheral blood in patients with locally advanced cervical cancer chemotherapy. Health Way, 14, 157-158.

Moher, D., Liberati, A., Tetzlaff, J., \& Altman, D. G. (2009). Preferred reporting items for systematic reviews and meta-analyses: the PRISMA statement. BMJ, 339(1), b2535. http://dx.doi.org/10.1136/ bmj.b2535. PMid:19622551.

Nemunaitis, J., Rabinowe, S. N., Singer, J. W., Bierman, P. J., Vose, J. M., Freedman, A. S., Onetto, N., Gillis, S., Oette, D., Gold, M., Buckner, C. D., Hansen, J. A., Ritz, J., Appelbaum, F. R., Armitage, J. O., \& Nadler, L. M. (1991). Recombinant granulocyte-macrophage colony-stimulating factor after autologous bone marrow transplantation for lymphoid cancer. The New England Journal of Medicine, 324(25), 1773-1778. http://dx.doi.org/10.1056/NEJM199106203242504. PMid:1903847.

Nie, J., Zhao, C., Deng, L., Chen, J., Yu, B., Wu, X., Pang, P., \& Chen, X. (2016). Efficacy of traditional Chinese medicine in treating cancer. Biomedical Reports, 4(1), 3-14. http://dx.doi.org/10.3892/ br.2015.537. PMid:26870326.

Pan, D. Z., \& Chen, L. Y. (2001). Clinical significance of medicated diet on improving nutritional status of patients with cancer chemotherapy. Dietotherapy and Herbal Med Diet, 6, 2-3.

People's Republic of China, Department of Health Management. (1991). Guidelines for the diagnosis and treatment of cancer. Beijing: Pecking Union Medical College Press.

Qu, D. (2018). Clinical efficacy of traditional Chinese medicine diet intervention on gastrointestinal reactions in patients with cancer chemotherapy. Home Med, 4, 229.

Shi, Y. F., Zhang, H. J., \& Han, C. R. (2012). Observation and prospect of dietetic nursing for Wuhong decoction in preventing and treating chemotherapy myelo suppression of breast cancer. China's Naturoathy, 20, 76-77.

Wang, H. J., Wang, Z. X., Guo, Y. D., Huang, Q. Z., Zhang, P. J., Chang, J. J., \& Zhang, Z. (2011). Clinical research on treating advanced breast cancer by righting training thanh hoa soup(bulk)with common herbs. Clinical Journal of Chinese Medicine, 3, 67-69.

Wang, Z. X., Wang, H. X., Guo, Y. D., Zhang, P. J., Chang, J. J., Huang, Q. Z., \& Zhang, Z.(2012). Treatment of 58 cases of common malignant tumors in the lower abdomen with Fuzheng Peibing Qinghua Decoction (San) combined with medicated diet. TCM Research, 25, 20-23.

Wu, Q. (2016). Observation on the application effect of traditional Chinese medicine diet in the rehabilitation of cancer patients. Cardiovascular Disease Journal of Integrated Traditional Chinese and Western Medicine, 4, 160.

Wu, Q., \& Liang, X. (2018). Food therapy and medical diet therapy of Traditional Chinese Medicine. Clinical Nutrition Experimental, 18, 1-5. http://dx.doi.org/10.1016/j.yclnex.2018.01.001.

Yue, L. Q., Peng, X. L., Yang, X. X., \& Liu, F. D. (2007). The effect of herbal - meals on peripheral blood cells for patients with gastric cancer receiving adjuvant chemotherapy. Chinese Journal of Modern Nursing, 26, 1241-1242.

Zhang, D. D., Wu, T., \& Mao, H. H. (2014). Investigate postoperative patients with head and neck cancer medicated diet therapy assistant clinical efficacy. Chinese Journal of Medical Guide, 16, 1127-1128. 
Zhang, W. X. (2009). Effect of diet therapy on quality of life in patients with breast cancer undergoing chemotherapy. Chinese General Nursing, 7, 33-34.

Zhao, C. H., Yue, Y. Z., Zhou, S. M., \& Yang, Y. F. (2017). Clinical observation on traditional chinese medicine dietary therapy for improving symptoms of cancer patients with chemotherapy. Modernization of Traditional Chinese Medicine, 4, 663-668.
Zheng, X., Chen, J., Xie, T., Xia, Z., Loo, W. T. Y., Lao, L., You, J., Yang, J., Tsui, K., Mo, F., \& Gao, F. (2017). Relationship between Chinese medicine dietary patterns and the incidence of breast cancer in Chinese women in Hong Kong: a retrospective cross-sectional survey. Chinese Medicine, 12(1), 17. http://dx.doi.org/10.1186/ s13020-017-0138-9. PMid:28670332. 